\title{
The Relationship Between Galactic Cosmic Rays and Solar Wind
}

\author{
Grace D. Ihongo* \\ University of Aberdeen, $U K$ \\ E-mail: g.ihongo@abdn.ac.uk
}

\section{Charles H T. Wang}

University of Aberdeen

E-mail: c.wang@abdn.ac.uk

\begin{abstract}
The relationship between galactic cosmic rays and solar wind is investigated using an extended time- dependent and anisotropic force field model, where galactic cosmic rays flux is found to be related to the solar wind speed through the local interstellar spectrum and a modulation parameter. Galactic cosmic ray flux calculated at $1 \mathrm{AU}$ within the energy range $(0.2-88) \mathrm{GeV}$ using the model is also presented, the flux variation with time calculated at $1 A U$ at a fixed energy range using the model is also presented and the solution is used to predict the flux variation at earth. The mechanism of cosmic rays transport considered here are only the diffusion and convection, other processes such as particle drifts, energy losses and magnetic irregularities among others are not considered in this work.
\end{abstract}

The 34th International Cosmic Ray Conference,

30 July- 6 August, 2015

The Hague, The Netherlands

\footnotetext{
*Speaker.
} 


\section{Introduction}

Despite the efforts of several researchers to explain cosmic rays' transport and its relationship with the solar wind, there are still fundamental uncertainties on the subject. A detailed study of the relationship requires many processes and different approximations of the well-known Parker transport equation[1,2]. These include but are not limited to convection in the solar wind, perpendicular diffusion, pitch angle diffusion, particle drifts due to heliospheric magnetic field lines, adiabatic energy losses and gains [2]. Although, this usually requires a numerical approximation of the transport equation since it is a complex process, however if only the diffusion and convective processes are considered, it can be modeled analytically as in the force field and convection diffusion approximations $[1,2,3]$

This paper studies extensively, the relationship between galactic cosmic rays and solar wind including its flux variation with time for a period of 30 days at moderately high energies using a time-dependent and anisotropic force field model. The flux variation is seen to be anti-correlated with the solar wind which is in good agreement with ground based Neutron monitors count rates. This is also in line with the proven anti-correlated cosmic rays relationship with solar activity [4]

Our model considers only diffusion and convective processes. Other processes mentioned above are beyond the scope of the model. The only type of cosmic rays considered here are the galactic cosmic ray protons. Other types of cosmic rays are beyond the scope of this study.

\section{The Model}

Figure 1: Model Presentation showing cosmic rays from outside the heliosphere

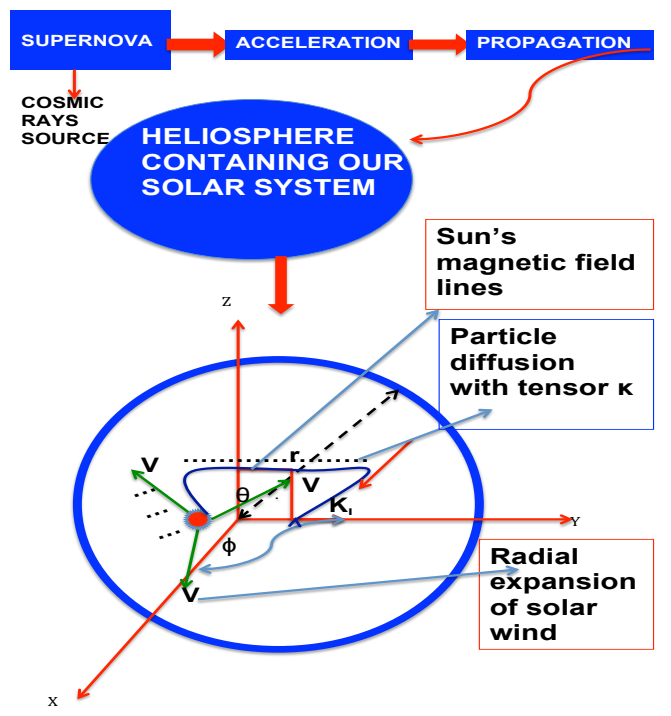


A time-dependent and anisotropic force field model is used to model galactic cosmic ray flux variation at $1 A U$.

Figure 1 presents the background idea of the model. As seen from the figure:

- Galactic cosmic rays enters the heliosphere

- Are trapped by the solar wind

- they interact with solar wind.

- The nature of interaction is through magnetic trapping as this is a macroscopic modeling.

- Only interaction and transport in the heliosphere are considered.

- Acceleration from source and propagation through the interstellar is beyond the scope of the model.

From fig $1, V$ is the solar wind speed, $\kappa$ is the diffusion coefficient. If particle's distribution is denoted by " $f$ " where $f=f(\mathbf{r}, p, t)$ then, the Parker's transport equation relating the above parameters is written in its simplest form as $[1,2,5]$

$$
\frac{\partial f(\boldsymbol{r}, p, t)}{\partial t}+\nabla \cdot \boldsymbol{S}-\underbrace{\frac{1}{3} \nabla \cdot \boldsymbol{V} \frac{\partial f(\boldsymbol{r}, p, t)}{\partial \ln p}}_{Q}=0
$$

where $\boldsymbol{S}=4 \pi p^{2}(\boldsymbol{C} \boldsymbol{V} f(\mathbf{r}, p, t)-\boldsymbol{\kappa} \cdot \nabla f(\mathbf{r}, p, t))$

Is the differential current density and $C=\frac{-1}{3} \frac{\partial \ln f}{\partial \ln p}$ Is the Compton-Getting factor, $p$ is the momentum and $Q$ is the term elucidating energy losses.

Eqn (1) have been solved using the following assumptions:

- There is a quasi-Stationary state such that $\frac{\partial f}{\partial t}=0$ and no sources

- There are no energy losses such that $\nabla \cdot V=0$ for $r \geq 1 A U$

- Galactic cosmic rays are carried by the solar wind

- The solar wind is radially dependent

- No particle drifts

- There is a small anisotropy in the solar wind such that $V=V(r, \theta, \tau)$

- The heliosphere is axially-symmetric in the heliocentric coordinate system where $\theta=\frac{\pi}{2}$

- Isotropic and parallel diffusion coefficient

- Perpendicular diffusion is negligible 
- Earth's velocity influence is negligible compared to solar wind speed.

When the above assumptions are applied to eq (1), it yields the extended time-dependent and anisotropic force field equation as

$$
\frac{\partial f(r, \theta, p, \tau)}{\partial r}+\frac{P V(r, \theta, \tau)}{3 \kappa} \frac{\partial f(r, \theta, p, \tau)}{\partial P}=0
$$

Where $V$ is the solar wind speed, $\kappa$ is the diffusion coefficient, $p$ is the momentum $\tau$ is the solar wind time. It is transformed from the normal time $t$ to the solar wind frame (Note that detailed transformation equations are omitted here due to the restricted length of the paper).

Equation 3 has been solved analytically which yields the following analytical solution in term of an arbitrary constant $F$ where $F$ is an arbitrary function to be fixed using the values at the boundary of the heliosphere.

$$
f(r, \theta, p, \tau)=F(\theta, \underbrace{-\int \frac{V(r, \theta, \tau)}{\kappa_{1}(r)} \mathrm{d} r+3 \int \frac{\beta \kappa_{2}(p)}{p} \mathrm{~d} p}_{X})
$$

where $X$ is assumed to be a constant such that the general solution is

$$
f(r, p, \theta, \tau)=F(\theta, X)
$$

Resulting to this specific solution

$$
\int_{p}^{p^{*}} \frac{\beta \kappa_{2}\left(p^{\prime}\right)}{p^{\prime}} \mathrm{d} p^{\prime}=\int_{r}^{R} \frac{V(r, \theta, \tau)}{3 \kappa_{0}} \mathrm{~d} r \equiv \phi(r, \theta, \tau)
$$

which yields

$$
\phi(r, \theta, \tau)=\int_{r}^{R} \frac{V(r, \theta, \tau)}{3 \kappa_{0}} \mathrm{~d} r
$$




\section{Model Solution}

The relationship between galactic cosmic rays and solar wind including cosmic ray transport is better understood using its spectrum as a function of its flux and the kinetic energy or rigidity.

Therefore, the observed cosmic ray flux spectrum with respect to rigidity is $[3,6]$

$$
J(p)=p^{2} f(p)
$$

where

$J(p)$ is the observed cosmic ray flux spectrum, $p$ is the rigidity of cosmic ray particles and $f$ is the omnidirectional distribution function of cosmic ray intensity.

Here, we convert the above spectrum to kinetic using the relation:

$$
\left(\frac{A}{Z}\right) \sqrt{T\left(T+2 T_{0}\right)}
$$

Where $A$ and $Z$ are the mass and atomic numbers of protons respectively.

This yields the model solution in terms of flux and kinetic energy as

$$
j(r, \theta, T)=j_{T^{*}}\left(\frac{T\left(T+2 T_{0}\right)}{T\left(T+2 T_{0}\right)+2\left(\sqrt{T\left(T+2 T_{0}\right)}\right) \Phi(r, \theta, \tau)+\Phi^{2}(r, \theta, \tau)}\right)
$$

where, $\Phi=\frac{Z e \phi(r, \theta, \tau)}{A}$ and $\phi(r, \theta, \tau)=\frac{V(r, \theta, \tau)}{3 \kappa_{0}}(R-r), A$ is the atomic mass, $Z$ is the atomic number of cosmic rays protons and $e$ is the electronic charge $. J_{T^{*}}\left(R, T^{*}\right)=b \beta\left(\frac{A}{Z e}\left(\sqrt{T^{*}\left(T^{*}+2 T_{0}\right)}\right)^{-a}\right.$ where $b$ and $a$ are constants and $\beta=1$.

$T$ is the kinetic energy and $T_{0}$ is the rest mass of a proton.

$*$ is used to denote the values at the boundary of the heliosphere.

Fig 2-3 shows the fit results and the modulation parameter $\phi$ which depends both $V$ and $t$ as shown in figure(4)

As seen from fig2, each solar wind ray travel at a particular speed and a given time thus each line in the figure on the left panel represents a solar wind ray with its speed at a given time $\tau$. The time and speed of each ray on the average is constant.

The right panel represent a solar wind ray with speed selected at a time $\tau=18 \mathrm{hrs}$ compared with the CAPRICE1994 and BESS experimental data. The model is consistent with data points within the energy range $(0.3-1.3) \mathrm{GeV}$. This set of data suggests that this model works best at energy range of approximately $1 \mathrm{GeV}$. 
Figure 2: Time-dependent and Anisotropic Force Field Solution left panel, Right panel is the model compared to data: The red cross is this work, the blue multiplication sign is CAPRICE1994 [7] and the green circle is BESS [8] . The fit is done for: $T_{0}=0.9384$; $G e V, R=100 A U, r=1 A U, \theta=$ $\frac{\pi}{2}, a=2.788, b=1.82 e 4, \kappa=7.3 \times 10^{20} \beta \mathrm{Pcm}^{2}(G V) / s$. Solar wind data are from ACE website: srl.caltech.edu/ACE/ASC/rtsw.html, and the black line is the local interstellar or the boundary spectrum. Note that the boundary spectrum is multiplied by a correction factor of $10^{2}$ to correct its uncertainties.
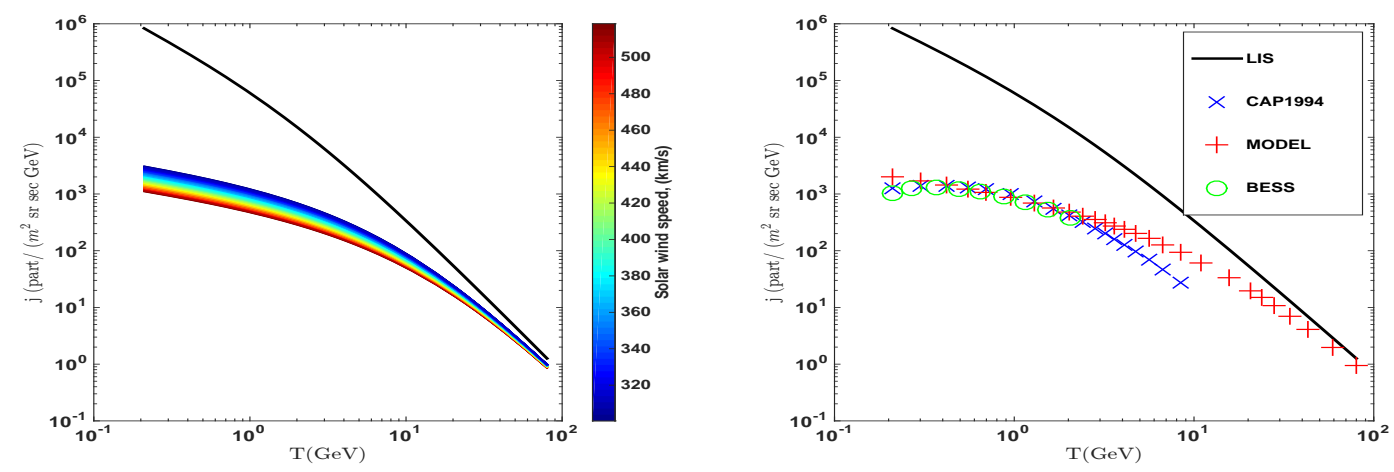

Figure 3: Calculated proton flux variation with time at a fixed energy range showing the relationship of GCR cosmic ray flux variation and solar wind; this is compared with data from two neutron monitors( top left is the model, left bottom 2 are counts are from Moscow NM: cro.izmiran.rssi.ru/mosc/main.htm and Oulu NM: cosmicrays.oulu.fi respectively). The fit is done for: $T_{0}=0.9384 \mathrm{GeV}, R=100 \mathrm{AU}, r=$ $1 A U, \theta=\frac{\pi}{2}, a=2.788, b=1.82 e 4, \kappa=7.3 \times 10^{20} \mathrm{~cm}^{2}(G V) / s$. Solar wind data are from ACE website: srl.caltech.edu/ACE/ASC/rtsw.html and right panel is the dependence of the modulation parameter on time. Note that the boundary spectrum is multiplied by a correction factor of $10^{2}$ to correct its uncertainties.
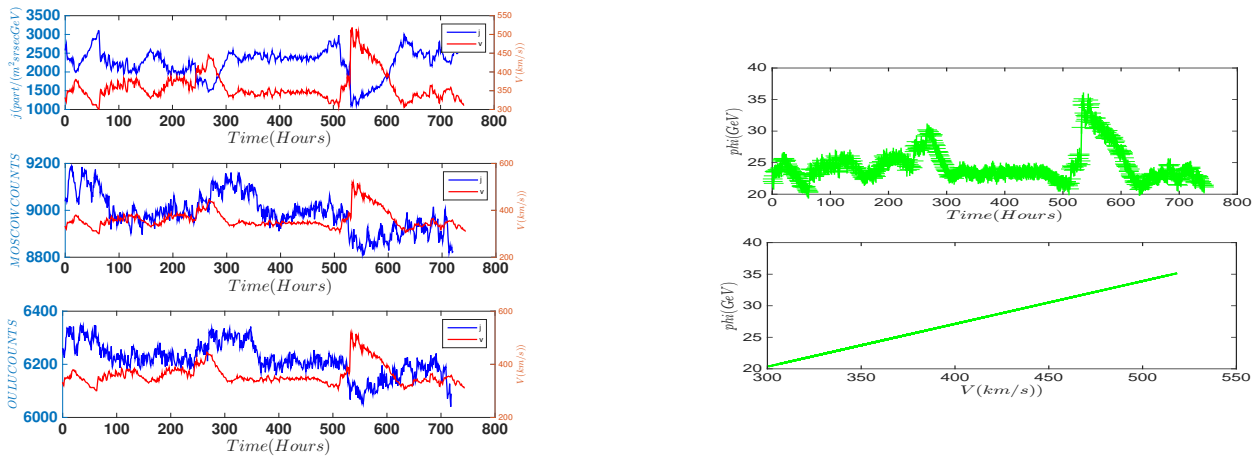

\section{Discussion and Summary}

Fig 2 shows galactic cosmic ray flux calculated a $1 A U$ using the model, it can be seen clearly that the spectrum bends over at low energies which is a clear evidence of the flux modulation by solar wind. The solar wind also cause variations in flux as each solar wind speed produces slightly a different spectrum . Note that the lines are very close to each other due the data used as they represent the data. 
Fig 3 presents a clear picture of the flux variation with time at a fixed energy range, the GCR flux is observed to be anti-correlated with the solar wind which is consistent with data from neutron monitors as seen on the last two panels. This means that periods of high solar wind on earth would record low cosmic ray flux and vice-versa, this also imply that GCR intensity variation on earth is dependent of the solar wind.

The right panel on fig 3 presents the behavior of the model's main modulation parameter " $\phi$ ", it varies with time between $(20$ and 35$) \mathrm{GeV}$ and increases with increasing solar wind. This means that the modulation parameter is inversely related to the calculated flux at $1 A U$ and has direct relationship with solar wind and solar activity. In summary, we can say that galactic cosmic rays and solar wind has the following possible relationship;

- Galactic cosmic ray flux variation and solar wind are anti-correlated at $1 A U$ as seen in fig 3.

- The flux variation at $1 A U$ is caused by the anisotropy in solar wind as seen in fig 2

- Galactic cosmic rays at moderately high energies are modulated by the solar wind at $1 A U$.

- Galactic cosmic rays variation at earth is possibly dependent on the solar wind. 


\section{Acknowledgements}

We are grateful to the following people /groups for their useful contributions.

- Members of the QG2 University of Aberdeen, UK.

- H. Moraal, School of Physics, Potchefstroom University, Potchefstroom, 2520, South Africa.

- R. A. Caballero-Lopez, Institute for Physical Science and Technology, University of Maryland, College Park, MD 20742, USA

\section{References}

[1] Luis Miguel Mina Lopes Batalha (2012) Solar Modulation effects on Cosmic Rays (Modelization with force field approximation, $1 D, 2 D$ numerical approaches and characterization with AMS-02 proton fluxes) Instituto Superior Tecnico Universidade Tecnico de Lisboa.

[2] Caballero-Lopez R.A. and Moraal H.(2004) Limitations of the force equation to describe cosmic ray modulation. Journal of Astrophysical Res. vol 109,A01101, doi:10.1029/2003JA010098.

[3] Moraal.H (2013) Cosmic-Ray Modulation Equations Space Sci Rev 176 : 299 - 319.

[4] Tiwari B.K, Ghormare B.R, Shrivastava P.K and Tiwari D.P.(2014) Dependence of the Heliosphere and cosmic ray $(C R)$ on solar activity Res. J. Physical Sci. Vol 2(5), p.9.

[5] Parker, E. N. (1965), The passage of energetic particles through inter-planetary space, Planet. Space Sci., 13, 9.

[6] McDonald F.B. Caballero-Lopez R.A. and Moraal H. (2005) Termination shock and heliosheath effects on cosmic ray energy changes in the heliosphere 29th International Cosmic Ray Conference Pune 00, 101 ÂUU104.

[7] Boezio, P. Carlson et al (1999) The cosmic ray proton and helium spectra between 0.4 and $200 \mathrm{GeV}$ The Astrophysical journal 467-468.

[8] Anraku, K. et al (2002) Measurement of cosmic ray Hydrogen and Helium and their isotopic composition with the BESS experiment

The Astrophysical Journal 255-256. 